\title{
Evidence of contributory role of inflammation in patients with knee osteoarthritis
}

\author{
Nehal A.Zaki ${ }^{a}$,Tahia H. Saleem ${ }^{b}$, Elsayed Said ${ }^{c}$,Mohammed H. Hassan ${ }^{a}$ \\ ${ }^{a}$ Department of Medical Biochemistry, Faculty of Medicine, South Valley University, Qena, \\ Egypt. \\ ${ }^{\mathrm{b}}$ Department of Medical Biochemistry, Faculty of Medicine, Assiut University, Assiut, Egypt. \\ ${ }^{c}$ Department of OrthopaedicSurgery, Faculty of Medicine, South Valley University, Qena, \\ Egypt.
}

\begin{abstract}
:
Background:Osteoarthritis (OA) is a debilitating degenerative joint disease particularly affecting weightbearing joints within the body, principally the hips and knees.

Objectives: We aimed to asses the inflammatory markers in the form of C-reactive protein (CRP) and erythrocyte sedimentation rate (ESR) among patients with knee OA.

Patients and Method: The study included 90patients with knee OA, 30 patients with mild degree of OA\&30 patients with moderate degree of OA \&30 patients with severe degree of OA. CRP and ESR wereperformed and analyzed for all the included patients.

Results: the mean CRP of all studied patients was $5.1 \pm 1.33 \mathrm{mg} / \mathrm{L}$ with minimum CRP value of 3 $\mathrm{mg} / \mathrm{dL}$ and maximum one of $7 \mathrm{mg} / \mathrm{dL}$. The mean ESR of all studied patients was $23.5 \pm 2.04 \mathrm{~mm} / \mathrm{h}$ with minimum ESR value of $20 \mathrm{~mm} / \mathrm{h}$ and maximum one of $27 \mathrm{~mm} / \mathrm{h}$. Statistically significant difference $(\mathrm{p}=$ 0.028 ) between mild OA \& severe OA groups as regard ESR, no other significant differences were observed.
\end{abstract}

Conclusions:The present study confirms the presence of inflammatory state among patients with knee osteoarthritis especially those with sever form.

Keywords: CRP, ESR, knee, Osteoarthritis.

\section{Introduction:}

Osteoarthritis (OA) is a degenerative joint disease characterized by articular cartilage degradation which can affect many joints in the body, but is particularly common in weight-bearing joints such as the knee and hip. The loss of cartilage can lead to joint space narrowing (JSN), pain, and loss of function and ultimately leads to the need for total joint replacement. There are a number of risk factors associated with OA, including genetic predisposition, obesity, age, and previous joint trauma. With obesity set to rise in future years, (Salihu et al., 2009) combined with OA being a frequent condition among the elderly and an ageing population (Mabey,Honsawek 2015), the prevalence of OA is expected to increase. An effective and reliable method for diagnosis and prognosis is needed, with increased demands on health services around the world.Radiography is routinely used to aid in the diagnosis of OA.However, radiographic imaging is ineffective at detecting and monitoring the biochemical changes within joint tissue which can occur long before symptoms present(Swearingen et al.,2010).Different compounds may show different biochemical marker properties at different stages of the disease, reflecting the pathophysiological changes occurring within the joint tissue.(Lotz et al., 2013) Therefore, characterization of potential biomarkers is important to ensure their appropriate and optimal use.The future challenge in the management of knee OA is to discover early tools for diagnosis, progression and monitoring of the disease, and to find effective therapeutic interventions. Biomarkers are among 
possible tools. Since OA is related to inflammation, inflammatory biomarkers, such as C-reactive protein (CRP) and Erythrocyte Sedimentation Rate (ESR) have been used to determine such relationship (Fernandez-Cuadros et al., 2018).

The ideal scenario, in terms of biochemical markers of OA, would be to have a noninvasive, reliable and valid biochemical marker or cluster of markers that could be measured to aid in the diagnosis and predict the development of OA in patients at an early stage before the disease becomes symptomatic. The ability to reduce the long-term effects of the disease could considerably reduce the substantial socioeconomic costs ofOA(Ruiz D et al.,2013).

This study aims to determine wheatehr there is a role of the inflammation in the pathogenesis of knee OA or not, via evaluation of CRP and ESR) in patients with various severities of knee OA.

\section{Materials\&Methods:}

- Study design: Aprospective, hospital based study carried out on 90 patients with knee osteoartheits of whom; 30 patients with mild OA, 30 patients with moderate OA \& 30 patients with severe OA. They wererecruited fromorthopaedic Surgery Department,Qena University Hospital, Egypt,during the period from February to July 2019.

\section{- Methodology:}

$5 \mathrm{ml}$ venous blood sample was collected from all included patients and divided into two parts; $3 \mathrm{mls}$ were evacuated into plain tubes for CRP assay and $2 \mathrm{ml}$ was placed into sodium citrate containing tubes for ESR measurements. For CRP assay, the blood samples were allowed to be clotted at room temprature for $20 \mathrm{~min}$ and then were centrifugated at 3000 rpmfor 10 minutes. The separated sera were used for
CRP assays immediately by the semiquantitative latex agglutination test (AVITEX CRP KITS: Catalog NO.OD023: supplied by Omega Diagnostics.UK). Antibodies to human CRP coat the AVITEX latex particles: when the latex suspension was mixed with the serum containing high CRP levels on a slide. Agglutination was seen within 2 min. and then serial dilutions of patient's serum in positive cases using isotonic saline were done to determine the CRP values. CRP consider normal if $<6 \mathrm{mg} / \mathrm{L}$. ESR assays were measured using (ESR STAT 6 Sed Rate Analyzer from HemaTechnologies, United States of America).ESR considered normal if $\leq 25 \mathrm{~mm} / \mathrm{h}$.

\section{Statistical analyses:}

Data were analyzed using Statistical Program for Social Science (SPSS) version 15.0. Quantitative data were expressed as mean \pm standard deviation (SD). Qualitative data were expressed as frequency and percentage.Independentsamples t-test of significance was used when comparing between two means. Level of significance was considered at $\mathrm{p}<0.05$.

Results:Demographic data: 90 patients including(10 males and 80 females),mean age of all studied patients was $45.5 \pm 10.6$ years.

Laboratory data:As regard CRP, the mean CRP of all studied patients was $5.1 \pm$ $1.33 \mathrm{mg} / \mathrm{L}$ with minimum CRP of $3 \mathrm{mg} / \mathrm{L}$ and maximum CRP of $7 \mathrm{mg} / \mathrm{L}$.

As regard ESR, the mean ESR of all studied patients was $23.5 \pm 2.04 \mathrm{~mm} / \mathrm{h}$ with minimum ESR of $20 \mathrm{~mm} / \mathrm{h}$ and maximum ESR of $27 \mathrm{~mm} / \mathrm{h}$. table (1).Statistically significant difference (pvalue $=\mathbf{0 . 0 2 8}$ ) between mild $\mathrm{OA} \&$ severe OA groups as regard ESR. Table (2). 
Table (1): values of CRP and ESR of the studied patients.

\begin{tabular}{|l|l|l|}
\hline \multicolumn{2}{|l|}{ Demographic \& Clinical data } & $\begin{array}{l}\text { Studied patients } \\
(\mathbf{N}=90)\end{array}$ \\
\hline \multirow{3}{*}{ CRP(mg/L) } & Mean \pm SD & $5.1 \pm 1.33$ \\
\cline { 2 - 3 } & Min - Max & $3-7$ \\
\hline \multirow{2}{*}{ ESR(mm/hour) } & Mean \pm SD & $23.5 \pm 2.04$ \\
\cline { 2 - 3 } & Min - Max & $20-27$ \\
\hline
\end{tabular}

Table (2): comparison of CRP and ESR among the studied patients as regard severity of OA.

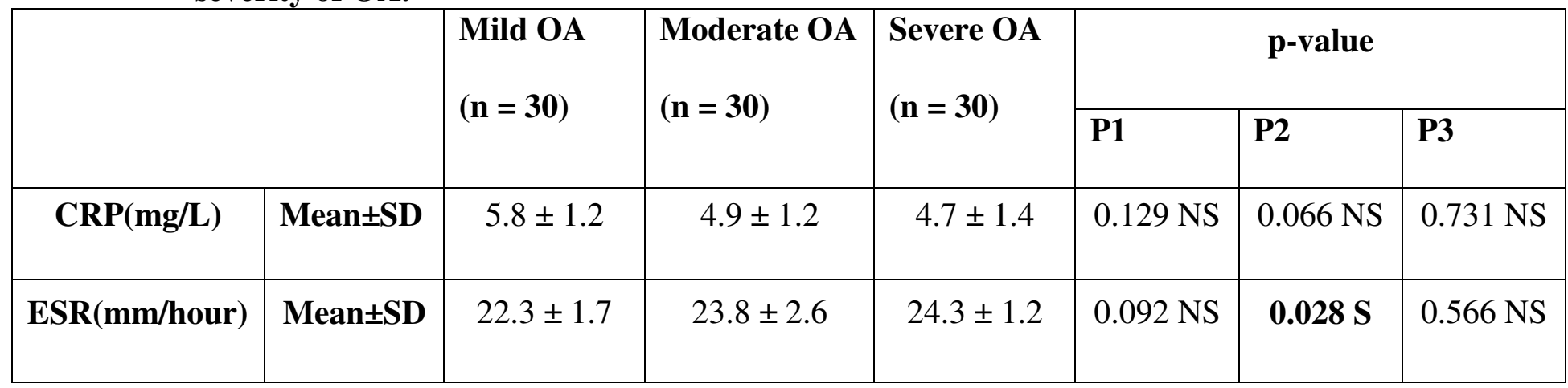

S: $p$-value $<0.05$ is considered significant.NS: $p$-value $>0.05$ is considered nonsignificant.

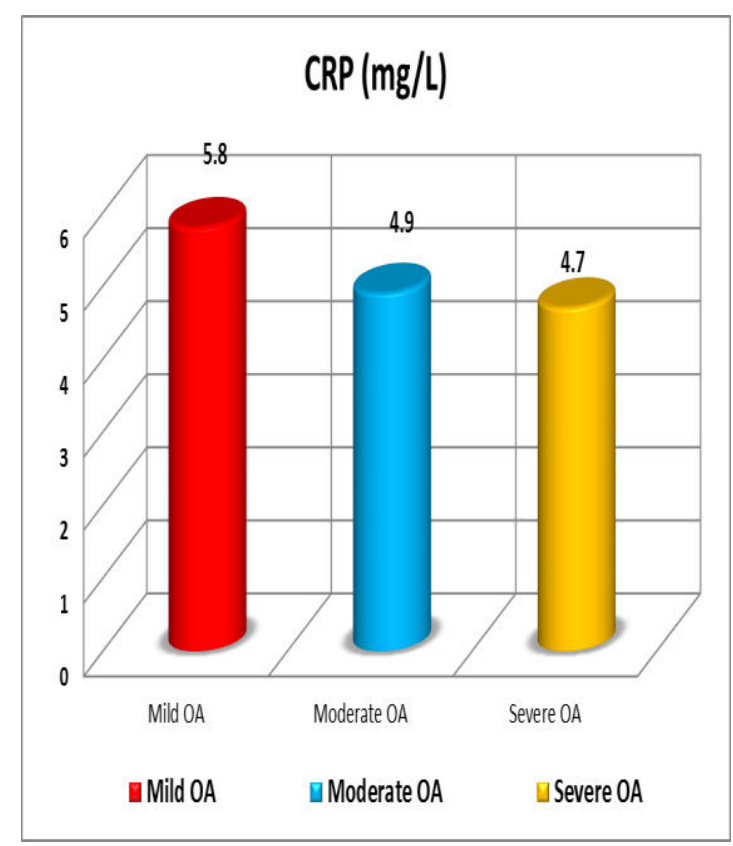

Figure (1): comparison of CRP as regard severity of $\mathrm{OA}$.

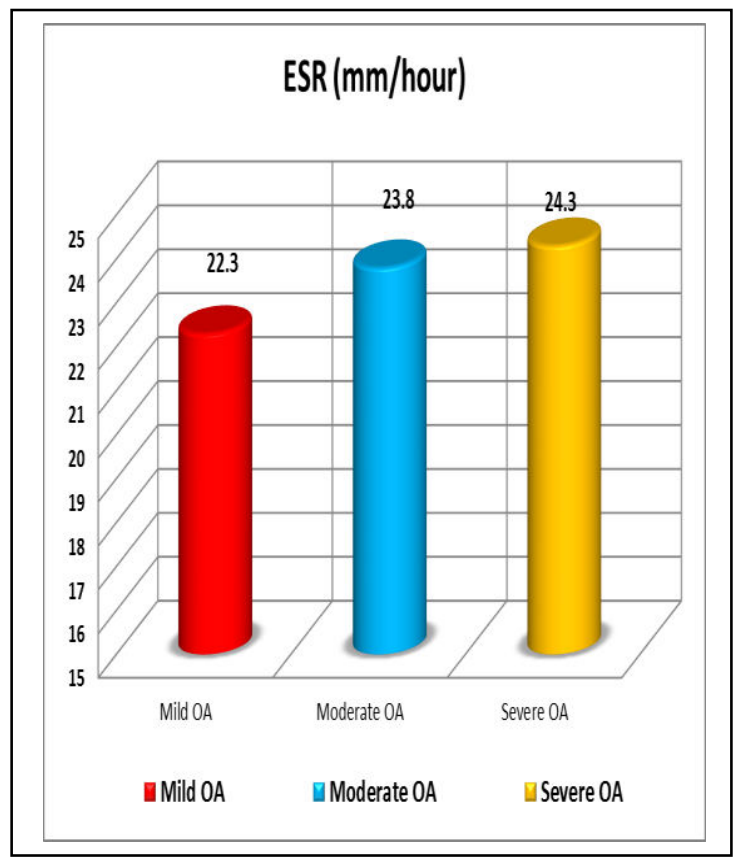

Figure (2): comparison of ESR as regard severity of $\mathrm{OA}$. 


\section{Discussion:}

Knee osteoarthritis (OA) is a major cause of pain and disability in subjects over 50 years with a significant impact on physical performance and quality of life (Conrozier et al.,2019).

Biomarkers of inflammation used in the study were CRP and ESR. These biomarkers are recognized as acute phase reactants or proteins. They indicate an inflammatory state, as in the case of rheumatic diseases. Erythrocyte Sedimentation Rate is cheap and commonly used in clinical practice. It mainly reflects fibrinogen concentration. Furthermore, CRP shows no variation with age, neither with erythrocytes morphology, nor with other protein variations. During inflammation, CRP concentration increases by 1000 folds; on the contrary, ESR increases by 2 to 5 folds. In an inflammatory process, such as orthopedic surgery, CRP reaches its maximum level at 24 to 48 hours and normalizes by the next week. On the contrary, ESR value is elevated after a week, and normalizes in the next 6 weeks (Möller I et al., 2016).

Several studies have observed that increased CRP levels are related to prevalence and progression of the knee or hip OA. With respect to OA, elevated CRP levels are related to synovial fluid IL-6 levels and to synovial infiltration, as well as with symptoms of pain and stiffness, radiographic grading, and disease progression (Smith et al., 2012).

\section{References:}

Conde J, Scotece M, Gomez R, Lopez V, Gomez-Reino, Gualillo O.et al., (2011).Adipokines and osteoarthritis: novel molecules involved in the pathogenesis and progression of disease, Arthritis, Vol. pg. 203901.
Many researchers worldwide have suggested that CRP and ESR are closely related to radiographic knee OA, symptoms severity, and the progression of the disease. These biomarkers suggest that low-grade inflammation might be a direct pathway in structural and symptomatic changes on knee OA (Zhu et al., 2016) In this study the mean of ESR for all studied patient was $22.3 \pm 1.7 \mathrm{~mm} / \mathrm{h}$ which was condiered as normal and the mean of CRP for all studied patients was $5.8 \pm 1.2 \mathrm{mg} / \mathrm{L}$ which also consider within normal range.

When considering the degree of OA severity, there was only Statistically significant difference between mild OA \& severe OA groups as regard ESR, otherwise all comparisons were statistically non-significant.

The results of the present study are in disagreement with Levinger et al., 2011; Conde et al., 2011;sanchezRamirez et al.,2014.On the other hand, Santos et al., 2011;Ferrucciet al., 2012; Xu et al., 2014did not find a significant correlation between inflammatory markers in plasma and knee osteoarthritis severity this is the same with our study result.

\section{Conclusion}

In conclusion, in patients with knee OA, levels of serum CRP and ESR were not associated with degree of severity of OA. Moreover, ESR and CRP provide no additional diagnostic accuracy in the diagnosis of knee osteoarthritis.

Conrozier T, Eymard F, Chouk M et al., (2019).Impact of obesity, structural severity and their combination on the efficacy of viscosupplementation in patients with knee osteoarthritis. BMC MusculoskeletDisord., 17: 20(1):376.

Ferrucci L, Penninx, Volpato et al., (2012). Change in muscle strength 
explains accelerated decline of physical function in older women with high interleukin-6 serum levels, J Am Geriatr Soc., Vol. 50 (pg. 1947-54).

Hame SL, Alexander RA.Knee osteoarthritis in women.Curr Rev Musculoskelet Med., (2013). Jun;6(2):182-7.

Levinger, Levinger, Trenerry MK, et al., (2011). Increased inflammatory cytokine expression in the vastuslateralis of patients with knee osteoarthritis, Arthritis Rheum, Vol. 63 (pg. 1343-8)

Lotz , Martel-Pelletier Christiansen C, Brandi ML, Bruyere O, Chapurlat $R$ et al.,(2013). Value of biomarkers in osteoarthritis: current status and perspectives. Ann Rheum Dis., 72(11):1756-63.

MabeyT,Honsawek

S. (2015).Cytokines as biochemical markers for knee osteoarthritis. World journal of orthopedics, 6(1): 95-105.

Marcos EFC, Olga Susana PM,Albaladejo-FlorinMJ,Ruben

AL. (2018). Ozone Decreases Biomarkers of Inflamation (C-Reactive Protein and Erytrocyte Sedimentation Rate) and Improves Pain, Function and Quality of Life in Knee Osteoarthrtitis Patients: A Before-and-After Study and Review of the Literature. Middle East J Rehabil Health Stud., In Press(In Press):e64507.

Möller I, Gharbi M, Martínez H, Herrero M, Vergés J, Henrotin Y et al.,(2016). Effect of chondroitin sulfate on soluble biomarkers of osteoarthritis: How to analyze and interpret the results from an open-label trial in unilateral knee osteoarthritis patients. OsteoarthrCartil., 24:77.

Powell A, Teichtahl AJ, Wluka AE, et al.,(2005). Obesity: a preventable risk factor for large joint osteoarthritis which may act through biomechanical factors. Br J Sports Med., Jan;39(1):45 .
Ruiz D, Koenig L, Dall TM, Gallo P, Narzikul A, Parvizi J et al., (2013). The direct and indirect costs to society of treatment for end-stage knee osteoarthritis. J Bone Joint Surg Am., 95:1473-1480.

Salihu HM, Bonnema SM, Alio AP.(2009). Obesity: What is an elderly population growing into? Maturitas., 63:7-12.

Sanchez-Ramirez, DC, van der Leeden, van der Eschet al.,(2014).Elevated C-reactive protein is associated with lower increase in knee muscle strength in patients with knee osteoarthritis: a 2-year follow-up study in the Amsterdam Osteoarthritis (AMS-OA) cohort. Arthritis

Res.Ther., 16, R123

Santos ML, Gomes WF, Pereira DS, et al., (2011). Muscle strength, muscle balance, physical function and plasma interleukin-6 (IL-6) levels in elderly women with knee osteoarthritis (OA), Arch GerontolGeriatr, Vol. 52 (pg. 322-6).

Smith JW, Martins TB, Gopez E, Johnson T, Hill HR, Rosenberg TD et al.,(2012). Significance of Creactive protein in osteoarthritis and total knee arthroplasty outcomes. TherAdvMusculoskelet Dis., 4(5):31525.

Swearingen CA, Carpenter JW, Siegel R, Brittain IJ, Dotzlaf J, Durham TB, Toth JL, Laska DA, Marimuthu J, Liu C, et al. (2010). Development of a novel clinical biomarker assay to detect and quantify aggrecanase-generated aggrecan fragments in human synovial fluid, serum and urine. Osteoarthritis Cartilage, 18:1150-1158.

Xu C, Guo H, Qu P, Fu J, Kuo FC, Chen JY. (2018). Preoperatively elevated serum inflammatory markers increase the risk of periprosthetic joint infection following total knee arthroplasty in patients with 
osteoarthritis. Therapeutics and clinical risk management, 14: 1719-1724.

Zhu, Jin, Wang B, Wluka A, Antony B, Laslett LLet al.,(2016).CrossSectional and Longitudinal Associations Between Serum Levels of High-Sensitivity CReactive Protein, Knee Bone Marrow Lesions, and Knee Pain in Patients With Knee Osteoarthritis. Arthritis Care Res (Hoboken), 68(10):1471-7. 